\title{
Towards a Web-Based Framework for Computer-Aided Manufacturing
}

\author{
Andrei Lobov ${ }^{1,2}$, Antti Martikkala ${ }^{1}$, Prasun Biswas ${ }^{1}$ \\ ${ }^{1}$ Automation Technology and Mechanical Engineering, Tampere University, Tampere, Finland \\ E-mail: \{andrei.lobov, antti.martikkala,prasun.biswas\}@tuni.fi \\ ${ }^{2}$ Mechanical and Industrial Engineering, Norwegian University of Science and Technology, Trondheim, Norway \\ E-mail: andrei.lobov@ntnu.no
}

Received: July 24, 2019

\begin{abstract}
Computer-aided manufacturing (CAM) helps to see how manufacturing processes could be executed checking if desired products can be made and at what cost. Usually, a 3D model of a targeted product is used as one of the inputs for CAM. Although actively used for some application domains and despite of well-developed CAM technologies and tools frameworks, further acceptance of CAM is hindered by, on one hand, a restricted access and, on another, knowledge needed to use CAM tools and methods. This article outlines a concept for web-based integration framework architecture for CAM. It defines main stakeholders and their roles in efficient product development and virtual commissioning.
\end{abstract}

Keywords: Computer-aided Manufacturing, Digital manufacturing, web tools, virtual commissioning

\section{INTRODUCTION}

With the development of Internet technologies and global connectivity of people the access to knowledge has simplified. The Wikipedia can be seen as an example of collective knowledge documenting and sharing. Those articles can be now accessed almost globally. Despite the questioning the quality or reliability of Wikipedia as a source of information, it is seen as "a valuable jumping off point for research" [1]. The Wikipedia, as any other encyclopedia, has limited ways to share the knowledge, as these mostly use text and images to systemically organize and describe selected concepts or phenomena in the articles. For the articles published on the Wikipedia, some basic animation and references to videos can be also added to further simplify presentation and comprehending of study materials.

The advent of Internet technologies pushed the capabilities of knowledge sharing further than just a passive perception from the paper or the screen. The study process can be organized now interactively. For example, massive open online courses or MOOC can integrate many people on the same platform with the tools helping to automate testing of students' knowledge (e.g. quizzes) and sometimes to interact with other students. The W3Schools [2], helping people to learn and apply web development standards, allows direct execution of code examples in a web browser to demonstrate their behavior. At the end, the leaner does not have to have any sophisticated tools, as a web browser could be enough.

(C) A. Lobov, A. Martikkala, P. Biswas, 2019 
Development of web technologies has affected also different engineering disciplines. In particular, for product design Computer-aided design (CAD) can be in principle and already done online. For instance, Onshape Product Design Platform [3] offers an interface to use $\mathrm{CAD}$ and related engineering APIs.

Industrial Internet can be defined as the application of Internet-based protocols and web technologies for industrial applications. The industrial applications differ from general 'office' applications in terms of requirements for reliability, availability and deterministic execution guaranteeing response times within certain time interval satisfying the needs of application. The use of Internet-inspired protocols can be found already at the production floor. For instance, EtherCAT [4], Ethernet/IP [5] and PROFINET [6] can be seen as examples of protocols, which appearance could be attributed to the success of the Internet.

This article describes a web-based framework that can support Computer-aided Manufacturing, so that engineers, on one hand, could benefit from integration of data at the different stages of product design and manufacturing, and, on the other, make it possible to integrate manufacturing system runtime data.

The rest of the paper is organized as follows. Next section describes the state of the art identifying the gaps and potential development directions. The state of the art is followed by a proposal of architecture for the framework to integrate engineering and runtime data. The fourth section contains the discussion followed by the conclusion and future work.

\section{STATE OF THE ART}

The problem of integration of various data can be approached in different ways. In [7], authors develop digital manufacturing toolbox aiming to assist, in particular, manufacturing SMEs, which may struggle to have sufficient resources for research, development and innovation (RDI) activates. That work defines digital manufacturing as tailored, case-specific integration of various technologies for the Production automation, Digital scanning and measurement, Manufacturing design and production simulation, Robotics, and, last, but not least additive manufacturing. Referred work does not suggest the use of web technologies rather proposing a selection of the tools via a process of discussion, preparatory work, tool process, delivery and customer care promoting a supervision of an expert from a particular organization.

Authors of [8] developed a system capable of monitoring main parameters of the CNC (Computer Numerical Control) equipment including the assessment of the consequences of the equipment failure. The proposed server/client architecture and the use of, for instance, "MS SQL Server2008 R2B" shows that the solution is build using Internet-based technologies integrating specific modules with Ethernet and Modbus protocols.

Prevention of confidentiality breach that could lead to the loss of Intellectual Property is discussed in [9]. As there are many cyber-physical or networked embedded systems start to serve the machine accessible with common internet protocols, these systems can be vulnerable points for hacker attacks. The authors of [9] look to the problem in a broader context rather than "simple" hacking of communication networks. They also consider, for example, dynamic response of a machine (e.g. 3D printer) in terms of vibrations produced that could be potential leakage of sensitive information about the work done by the machine. [10] has further elaborated various information leakage channels that in addition to vibration may include acoustic, power and magnetic side-channels. The mission is the development of information leakage-aware CAM tool.

Development of the Internet gave rise also to the new principles of software management. Online software package managers give developers a possibility to dynamically upload re- 
quired libraries from the Internet. One of such managers is the package installer for Python (pip) ${ }^{1}$. In [11] a Python-based 3-axis CNC plotter has been developed. The authors highlighted in their work among other things that their solution is following the principles of "open source, easy to understand, requiring basic programming knowledge, thereby making it easily amendable for the user" and "hardware which is less expensive and a setup which is less complex." In other words, both software and hardware for the CNC plotter are easy to develop and manage.

In [12] researchers have benefited from Open NX, "a collection of APIs that allows user to create custom applications for NX through an open architecture using well-known programming languages"2, developing CAM assistant program helping with tool-path planning and selection of cutting tools depending on materials in use. Same as in the abovementioned example of Python-based solution, here also the principle of developing own add-ons for a commercial tool is supported with openness of interface, dedicated APIs for known programming languages.

[13] have made a literature review for STEP-NC aiming to help with data integration between CAD-CAPP (Computer-aided Process Planning)-CAM-CNC. ISO 14649 (STEP NC), which is entitled as "Industrial automation systems and integration", format aims to provide bi-directional data flow between the manufacturing tools. It is according to [13] at the early stage with respect to its inclusion and active use with manufacturing systems. [14] proposes to use graph-based methods and algorithms to support data representation and analysis. Different types of graphs can be employed for corresponding problems ranging from product and process representative models to support of overall decision making and design of assembly lines. In [15], Visual C++ was used to develop "simple and affordable CAPP system". Here the simplicity and affordability can be attributed to the use of open formats, such as, for example, DXF (Drawing eXchange Format) originally developed for AutoCAD that can be also opened by many CAD applications.

[16] highlights the role of the Industrial Internet in integration of CAD-CAE (Computeraided Engineering)-CAPP-CAM allowing worldwide integration at the same time assuring product quality. Machines and the factories are seen as the nodes in the Industrial Internet in reference to the Industry 4.0 concept having web-based highly-automated smart factories. Such an integration may require end-to-end digitalization [17] supporting gradual development of production automation as a whole involving various information systems, such as Manufacturing Execution Systems (MES) and Enterprise Resource Planning (ERP). Furthermore, cloud-based architectures can be employed for the task of integration to develop so called cloud manufacturing environment [0]. In such an environment, the manufacturing services can be seen as independent entities capable of own decision making as these interact with the users.

In [19], a generic architecture for controlling Smart Manufacturing System (SMS) is described. The architecture includes a possibility for making new task plans, performing data analytics, diagnosis of disturbances to determine resilience strategy. The integration of SMS in the view of the authors should be supported via the use of cyber-physical systems (i.e. embedded networked systems) and revised concept of agents that may include "combinations of humans, cyber-, physical- and cyber-physical technologies" [19].

Another approach for integration can be found in [20] that outlined a Semantic Web Services framework, where different actors, e.g. device vendors, equipment vendors, system integrators, standardization institutions and customers cooperate using a common language -

\footnotetext{
${ }^{1}$ https://pypi.org/project/pip/

${ }^{2}$ https://docs.plm.automation.siemens.com/tdoc/nx/10/nx_api
} 
the Web Ontology Language $(\mathrm{OWL})^{1}$ - for building a manufacturing system. The ontologies can be organized into three main categories that of Product, Process and Resource (that also includes, for instance, equipment) [21]. The Process ontology can be seen as a pivotal point to join product and resource ontologies, i.e. to match product needs and skills/capabilities provided by resources.

\section{PROPOSED ARCHITECTURE}

Following the "triangle" of Product, Process, Resource outlined in the previous section, that can be extended for Computer-aided Manufacturing integrating necessary services and tools for going from the design of a product to its manufacturing. Fig. 1 presents overall integration framework, where the product and its needs can be depicted via the use of CAD applications, production equipment (manufacturing resources) should be considered via the CAM tools. These to be joined with valid process models, ideally supported by CAPP applications.

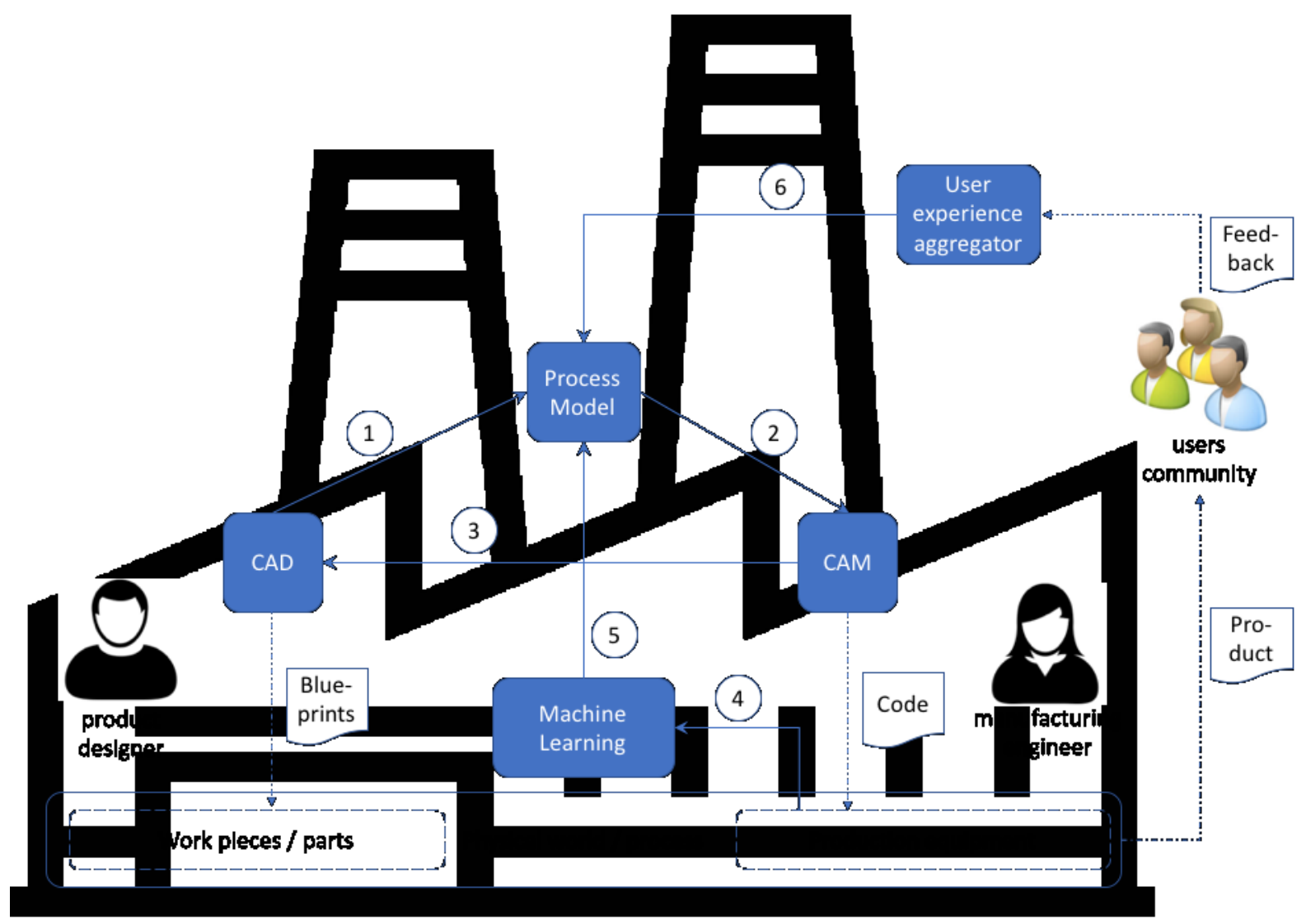

Figure 1. Integration Framework for Computer-aided Manufacturing

The process model block can be seen as a central element, because any inconsistencies during production process would signify that the loop (1)-(2)-(3) (the "triangle") has some deficiencies that have to be corrected. Thus, the analysis of possible corrective measures should start from the process model block.

\footnotetext{
${ }^{1}$ https://www.w3.org/OWL/
} 
In Fig. 1 the numbers on the edges are used to refine given relationships. The refinements are summarized in Table 1.

Table 1. Relations description in the proposed framework

\begin{tabular}{|c|c|}
\hline Relation \# & Description \\
\hline 1 & $\begin{array}{l}\text { Product designer inputs the CAD model representing a part or a workpiece to the CAPP } \\
\text { application for generating production plan taking into account the structure of a product, } \\
\text { requirements and materials it is made of. Selection of materials can be automated based } \\
\text { on the requirements the final product / workpiece should meet. }\end{array}$ \\
\hline 2 & $\begin{array}{l}\text { The process plan is input for the CAM tool that refines it in accordance to the available } \\
\text { equipment at the production floor. }\end{array}$ \\
\hline 3 & $\begin{array}{l}\text { As there could be some inconsistences noticed, these can be reported back to the prod- } \\
\text { uct designer by manufacturing engineer in order to try to find if there are possibilities to } \\
\text { change the design to meet product required and be able to manufacture the product. }\end{array}$ \\
\hline 4 & $\begin{array}{l}\text { As the product is being manufactured, the production equipment can report real-time } \\
\text { process data to learn on actual manufacturing system behavior. }\end{array}$ \\
\hline 5 & $\begin{array}{l}\text { The aggregated data from the actual process is used to fine tune process models. Thus, } \\
\text { an earlier feedback to the product designer could be possible based on manufacturing } \\
\text { site data. }\end{array}$ \\
\hline 6 & $\begin{array}{l}\text { As the products reach their final (or next stage) customers, there should be a possibility } \\
\text { to learn on customer's feedback, which is again fed to the process model, i.e. it is not } \\
\text { the task of designer or manufacturing engineer to analyze how the manufacturing site is } \\
\text { doing as a whole, but through the (adjusted) process models it should become evident } \\
\text { which particular requirement is not met or needs further improvement. }\end{array}$ \\
\hline Blueprints & $\begin{array}{l}\text { Workpieces / products / parts blueprints are generated with CAD tools and ideally } \\
\text { should accompany products through different stages of product lifecycle. }\end{array}$ \\
\hline Code & $\begin{array}{l}\text { Code for equipment (e.g. G-code for CNC machines) generated to produce correspond- } \\
\text { ing products. }\end{array}$ \\
\hline Product & Actual product that goes out of given manufacturing system to its customers. \\
\hline Feedback & $\begin{array}{l}\text { Customers' feedback is collected. It could be ranging from human-made reports to au- } \\
\text { tomated feedback collected via the IoT instruments connected to the product. }\end{array}$ \\
\hline
\end{tabular}

As the feedback received from users, it is processed in terms of process model and manufacturing resources before reaching product designer (e.g. CAD engineer). Thus, the designer is faced not only with direct customer feedback (which is important to know in any case), but also with the implications of the feedback for the other phases of product development and manufacturing.

Integration of abovementioned elements can happen via web technologies and, in particular, following Service-oriented Architecture (SOA). The SOA provides well-defined interfaces such as for example Web Service Description Language (WSDL), which possible application is described in [20]. Having common language representing services and its capabilities makes it possible for a computer to automatically find and integrate required modules for the application. Another approach for service definition is based on RESTful Web Services ${ }^{1}$, where the resource identification is based on the notion URI, i.e. Uniform Resource Identifier (everything in a way can be seen as a web resource, having dedicated unique address) with basic set of operations to create, read, update, delete (CRUD) online resources using HTTP (HyperText Transfer Protocol) ${ }^{2}$ methods PUT, GET, POST and DELETE.

\footnotetext{
${ }^{1}$ https://docs.oracle.com/javaee/6/tutorial/doc/gijqy.html

${ }^{2}$ https://tools.ietf.org/html/rfc2068
} 


\section{DISCUSSION}

There are already available ad hoc demonstrators for various steps outlined in Table 1. However, complete integration is still a challenging task partially due to the lack of agreement for common standards and protocols. Further development of Internet technologies is seen as a key to find a solution based on most commonly used communication protocols and knowledge representation formats. Similar, as for the growing share of Ethernet-based protocols for industrial automation, the same trend can help stabilizing web-based integration technologies for CAM.

Web-based integration framework for CAM can help developing and validating new products in SMEs having simple access to available and affordable modules and tools that may be already packaged for some widely-used (web) programming languages such as JavaScript (Node.js) or Python ${ }^{1}$.

In addition to the stakeholders explicitly listed in Fig. 1, there are developers of those open source modules and tools supporting CAD, CAM, machine learning and process definition algorithms. There probably would exist a gap in a quality and usability for some of the "free" modules. However, those are seen to diminish as the standards for service definition and descriptions will be further elaborated.

\section{CONCLUSION AND FUTURE WORK}

In this paper, we argue that web-based framework can be a solution for making wider use of Computer-aided Manufacturing. The web standards and technologies being extensively used, tested and developed for a large community of users are possible to apply in domain of manufacturing at different levels of the enterprise ranging from a production floor up to the ERP systems supporting strategic decision making. There are already first successful ad hoc applications available, although the challenge remains for finding widely acceptable solution. Such a solution is envisioned to appear as winning web standards for communication and knowledge representation get further developed and accepted.

\section{REFERENCES}

1. Reliability of Wikipedia. Retrieved July, 2019, from the Wikipedia website: https://en.wikipedia.org/wiki/ Reliability_of_Wikipedia

2. W3Schools.com: The World's Largest Web Developer Site. Retrieved July, 2019, from: https://www. w3schools.com

3. Onshape Product Design Platform. Retrieved July, 2019, from: https://www.onshape.com

4. EtherCAT Technology Group. Retrieved July, 2019, from: https://www.ethercat.org/default.htm

5. Ethernet/IP. Retrieved July, 2019, from ODVA website: https://www.odva.org/Technology-Standards/ EtherNet-IP/Overview

6. PROFINET Specification. Retrieved from PROFIBUS and PROFINET International website, July, 2019: https://www.profibus.com/download/profinet-specification

7. Kaartinen, H., Pieskä, S., \& Vähäsöyrinki, J. (2016). Digital manufacturing toolbox for supporting the manufacturing SMEs. In: 2016 7th IEEE International Conference on Cognitive Infocommunications (CogInfoCom) (pp. 000071-000076). Wroclaw, Poland: IEEE. doi: 10.1109/CogInfoCom.2016.7804527

8. Holopov, V., Kushnir, A., Kurnasov, E., Ganichev, A., \& Romanov, A. Development of digital production engineering monitoring system based on equipment state index. In: 2017 IEEE Conference of Russian

\footnotetext{
${ }^{1}$ https://github.com/aewallin/opencamlib
} 
Young Researchers in Electrical and Electronic Engineering (EIConRus) (pp. 863-868). St. Petersburg, Russia: IEEE. doi: 10.1109/EIConRus.2017.7910692

9. Chhetri, S. R., Faezi, S., \& Faruque, M. A. A. (2017). Fix the leak! an information leakage aware secured cyber-physical manufacturing system. In: Design, Automation \& Test in Europe Conference \& Exhibition (DATE), 2017 (pp. 1408-1413). Lausanne, Switzerland: IEEE. doi: 10.23919/DATE.2017.7927213

10. Chhetri, S. R., Faezi, S., \& Al Faruque, M. A. (2018). Information leakage-aware computer-aided cyberphysical manufacturing. In: IEEE Transactions on Information Forensics and Security, 13, 2333-2344. doi: 10.1109/TIFS.2018.2818659

11. Shivakumar, T., Sravan, M. S., \& Selvajyothi, K. (2016). Python based 3-Axis CNC plotter. In: 2016 IEEE International Conference on Power and Energy (PECon) (pp. 823-827). Melaka, Malaysia: IEEE. doi: 10.1109/PECON.2016.7951672

12. Chung, C., \& Ma, T. (2017). Implementation of CAM programming with machinability database. In: 2017 IEEE/SICE International Symposium on System Integration (SII) (pp. 236-240). Taipei, Taiwan: IEEE. doi: 10.1109/SII.2017.8279218

13. Ubaid, A. M., \& Dweiri, F. T. (2018). Sustainable systems integration by means of STEP-NC: Literature review. In: 2018 Advances in Science and Engineering Technology International Conferences (ASET) (pp.1-8). Abu Dhabi, United Arab Emirates: IEEE. doi: 10.1109/ICASET.2018.8376883

14. Weise, J., Benkhardt, S., \& Mostaghim, S. (2018). A survey on graph-based systems in manufacturing processes. In: 2018 IEEE Symposium Series on Computational Intelligence (SSCI) (pp. 112-119). Bangalore, India: IEEE. doi: 10.1109/SSCI.2018.8628683

15. Karim, M. S. A., \& Tai Tiong, C. (2019). Development of a simple and affordable computer aided process planning (CAPP). In: 2019 Advances in Science and Engineering Technology International Conferences (ASET) (pp. 1-4). Dubai, United Arab Emirates: IEEE. doi: 10.1109/ICASET.2019.8714443

16. Zhu, C., Zhao, S., \& Li, S. (2018). A study of small complicated axisymmetric parts manufacturing in industry 4.0. In: 2018 5th International Conference on Industrial Engineering and Applications (ICIEA) (pp. 158-162). Singapore, Singapore: IEEE. doi: 10.1109/IEA.2018.8387088

17. Oleynik, A. V., Kuznetsova, L. V., \& Petrov, V. E. (2018). Digital transformation of machine-building production. In: 2018 IEEE International Conference "Quality Management, Transport and Information Security, Information Technologies" (IT\&QM\&IS) (pp. 704-708). St. Petersburg, Russia: IEEE. doi: 10.1109/ITMQIS.2018.8524977

18. Lou, P., Zhu, C., Yan, J., Zhang, X., \& J. Hu. (2018). The emergence of cooperative behaviors under the incentive mechanism of profit allocation in a cloud manufacturing environment. In: 2018 rrd International Conference on Information Systems Engineering (ICISE) (pp. 147-151). Shanghai, China: IEEE. doi: 10.1109/ICISE.2018.00035

19. Romero, D., Jones, A. T., \& Wuest, T. (2018). A new architecture for controlling smart manufacturing systems. In: 2018 International Conference on Intelligent Systems (IS) (pp. 421-427). Funchal - Madeira, Portugal: IEEE. doi: 10.1109/IS.2018.8710540

20. Lobov, A., Lopez, F. U., Herrera, V. V., Puttonen, J. \& Lastra, J. L. M. (2008). Semantic Web Services framework for manufacturing industries. In: 2008 IEEE International Conference on Robotics and Biomimetics (pp. 2104-2108). Bangkok, Thailand: IEEE. doi: 10.1109/ROBIO.2009.4913327

21. Ferrer, B. R., Mohammed, W. M., Lobov, A., Galera, A. M., \& Lastra, J. L. M. (2017). Including human tasks as semantic resources in manufacturing ontology models. In: IECON 2017 - 43rd Annual Conference of the IEEE Industrial Electronics Society (pp. 3466-3473). Beijing, China: IEEE. doi: 10.1109/IECON.2017.8216587 